\title{
Finite-Precision Optimization of Time-Domain Digital Back Propagation by Inter-Symbol Interference Minimization
}

\author{
Christoffer Fougstedt ${ }^{(1)}$, Lars Svensson ${ }^{(1)}$, Mikael Mazur ${ }^{(2)}$, Magnus Karlsson ${ }^{(2)}$, and \\ Per Larsson-Edefors ${ }^{(1)}$
}

(1) Dept. of Computer Science and Engineering, Chalmers University of Technology

(2) Dept. of Microtechnology and Nanoscience, Chalmers University of Technology

Email: chrfou@chalmers.se

\begin{abstract}
We consider the effects of limited-resolution arithmetic on the performance of Time-Domain Digital Back Propagation. By minimizing resulting ISI of the quantized FIR impulse responses, we can achieve floating-point performance using 9-bit pairwise optimized filter coefficients.
\end{abstract}

\section{Introduction}

The Kerr non-linearity is one of the major reachlimiting factors in fiber-optic communication systems. While there have been significant research efforts on digital signal processing (DSP) nonlinear compensation techniques, such as digital back propagation (DBP), these simulations and experiments generally use floating-point off-line $\mathrm{DSP}^{2,4}$. Practical implementation aspects such as finite-precision effects, which are crucial due to the low-power and high-throughput requirements in real-time systems, remain largely unexplored.

To estimate the transmitted data, DBP emulates propagation through a fiber having negated parameters with respect to the forwardpropagation fiber. Thus, real-time implementation of DBP in an application-specific integrated circuit (ASIC) based on the repeated use of fast Fourier transforms would not only result in complex ASIC circuitry, but also introduce additional rounding errors due to the fixed-point representation required in ASICs. In contrast, since the dispersive steps are relatively short in DBP, we recently proposed to implement DBP in the time-domain with short finite impulse response (FIR) filters; the time-domain DBP algorithm (TD-DBP) ${ }^{1}$. In this previous work, we showed that correlated errors present a significant issue in DBP algorithms.

Here, we present TD-DBP finite-precision optimization, which uses co-optimization of consecutive filter pairs to minimize inter-symbol interference (ISI). The dispersive step dominates the power dissipation in our preliminary implementation studies (approx. 85\%), so optimization of the dispersive step is likely to significantly reduce overall power dissipation. By adjusting the amplitude of the impulse response, and thus how much of the available dynamic range is used, we generate several different quantized coefficient sets. We show that the pre-quantization amplitude correlates very poorly with bit-error rate (BER) performance, and introduce a novel metric-the signal-to-interference ratio (SIR)-which correlates well with BER performance $\left(R^{2}=0.842\right)$. We show that we can obtain close to floating-point performance using as few as 9 coefficient bits, giving a 30\% reach improvement at a BER of $10^{-3}$, in comparison to using individually-optimized coefficients.

\section{Time-Domain Digital Back Propagation}

Light propagation in optical fibers is described by the non-linear Schrödinger equation, which in simulations is generally solved numerically. In the commonly employed split-step Fourier method ${ }^{2}$, the non-linear and linear effects are treated as independent over a short propagation step in the fiber and can, thus, be split into one non-linear $(\hat{N})$ and one dispersive step $(\hat{D})$.

In this work, we use a two-step-per-span TDDBP algorithm (Fig. 1) with modified logarithmic distribution ${ }^{4}$ of the steps with the adjustment factor $k=0.4$. Each $\hat{D}$ uses a 17-tap least-squares constrained-optimization (LS-CO) FIR filter ${ }^{3}$ to compensate for accumulated chromatic dispersion (CD) corresponding to the step length, with the in-band response optimized with respect to the pulse-shaped spectrum.

\section{Finite-Precision Optimization}

Quantization-induced correlated errors adversely affect performance in fixed-point implementation of $\mathrm{DBP}^{1}$. Since DBP algorithms consist of many cascaded steps, correlated errors quickly build up, and thus, there is a significant potential for improving performance if the correlated errors can be minimized. In this work, we select a quan- 

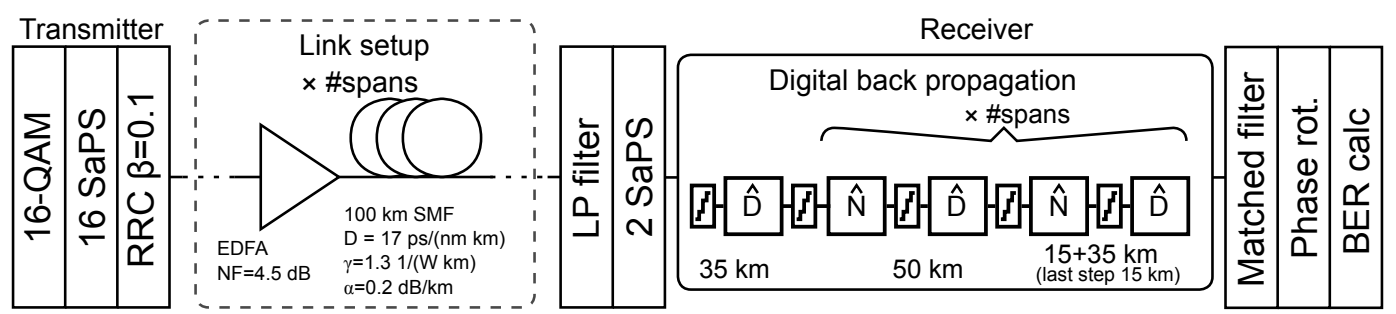

Fig. 1: Block diagram of the simulated system, with requantization steps shown in the DBP algorithm.

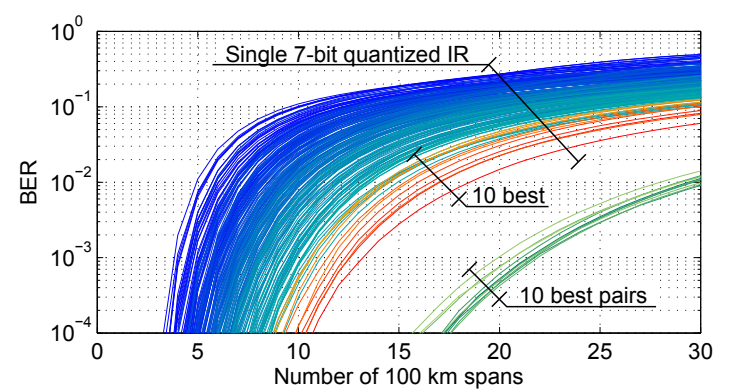

Fig. 2: BER as function of number of 100-km spans covered, for 217 IRs quantized to 7 bits. The ten best IRs according to the SIR metric have been highlighed in yellow/red. The figure also shows the performance of the ten best IR pairs according to the same metric.

tized filter based on minimizing the deviation of the resulting received and matched-filtered pulse shape from the time-domain Nyquist ISI criterion. We create a collection of quantized impulse responses (IRs) as follows:

- We first normalize the amplitude of the fullprecision IR to use the full range of quantized values at the desired resolution, and record the resulting quantized IR.

- We then gradually reduce the amplitude of the full-precision IR until a minimal change occurs in the quantized IR; record the quantized IR; and repeat these steps until the amplitude has been reduced by one half.

The process yields a collection of quantized IRs with slightly different amplitudes and slightly different sets of quantization errors. Compensation for the amplitude differences may be subsumed under inter-stage signal scaling; but the quantization errors affect performance significantly. Fig. 2 shows simulated BER vs number of 100 $\mathrm{km}$ spans covered for 217 IRs quantized to 7 bits, using the simulation setup described below. At a BER of $10^{-3}$, the reach ranges from less than 4 spans to more than 13 (more than a factor of 3 ).

Especially for the larger collections of IRs resulting from longer filters and higher resolutions, it is desirable to select a suitable IR without exhaustive system simulation as in Fig. 2. A reasonable assumption might be to prefer the highestamplitude IR in order to maximize the signal-toquantization-noise ratio (SQNR); but IR amplitude itself is a bad predictor of system performance.

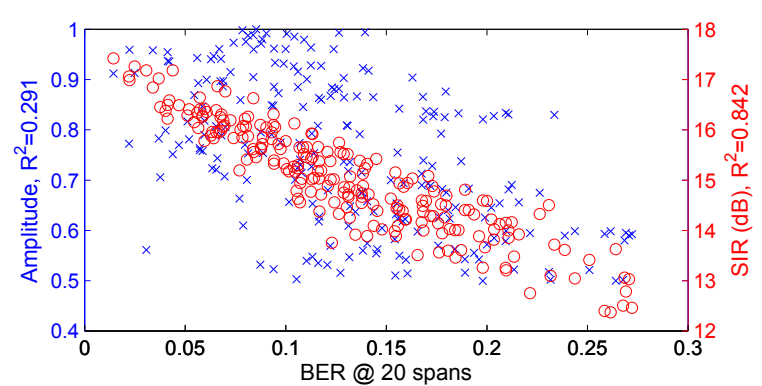

Fig. 3: BER at 20 100-km spans for each of the 217 7-bit IRs. The pre-quantization amplitude is shown in blue; the proposed SIR metric is shown in red.

Fig. 3 relates the BER at 20 spans with the IR amplitude for each of the 217 7-bit IRs; there are 39 IRs with higher amplitude than the bestperforming one. In the same figure, we also illustrate the metric used in the following: the ISI for a received signal, assuming root raised cosine (RRC) filters at transmitter and receiver sides and $C D$ according to one $50-\mathrm{km}$ fiber stage, expressed as the SIR in dB. In Fig. 2, we have highlighted the curves for the 10 "best" IRs according to this metric; these are gathered at the highperformance end of the range.

Even the small deviations caused by a wellchosen IR may combine to cause large overall errors when many filters are cascaded. Our two-steps-per-span architecture suggests a further optimization, where two different quantized IRs are used in each step to minimize the overall deviation from the real case. The number of 2-combinations grows quadratically with the number of quantized IRs, but the cheap ISI-based metric is trivially extended to the combined case, allowing the selection of promising candidates for simulation. Fig. 2 also shows the performance for the ten best IR pairs at the same resolution. The reach at $B E R=10^{-3}$ may be improved by more than $50 \%$ compared to the best single-IR case.

\section{TD-DBP Simulation Setup}

We use the same system setup as in our previous work ${ }^{1}$, i.e., a single-channel 16-QAM transmission. Our setup, which is shown in Fig. 1, uses the split-step Fourier method to simulate forward propagation with parameters as follows: 


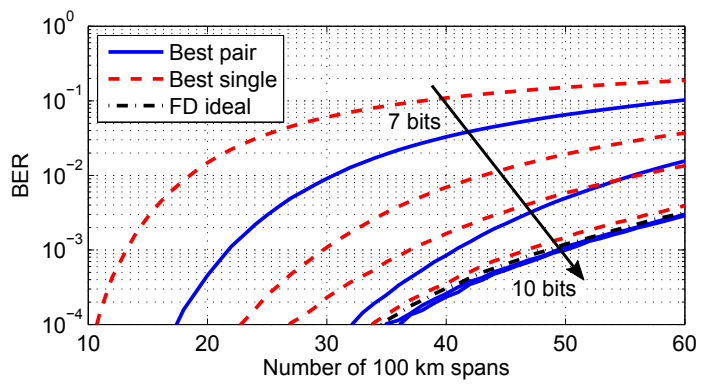

Fig. 4: BER as a function of 100-km spans for 7-10-bit coefficients, and the frequency-domain (FD) reference algorithm. Performance for 9- and 10-bit pairs are indistinguishable at this scale.

$\lambda=1550 \mathrm{~nm}, \mathrm{RRC}$ pulses at $20 \mathrm{GBd}, 16$ samplesper-symbol (SaPS), and 200 equally distributed steps-per-span. Each span consists of $100-\mathrm{km}$ fiber, and an EDFA compensating for the span loss, with a noise figure of $4.5 \mathrm{~dB}$. We sweep the number-of-spans parameter and record the BER for $2^{19}$ symbols per run. The optimal launch power was found to be approximately $1 \mathrm{dBm}$ when employing the 2-steps-per-span TD-DBP algorithm.

\section{Results: Impact on TD-DBP}

Following the procedure outline above, we generate collections of quantized IRs for each resolution (collection sizes double for each additional bit). Using the SIR-based metric, we select 10 top candidates at each resolution which are then simulated in order to find the best set. Fig. 4 shows the resulting BER as a function of number of spans for the coefficients with best in-simulation BER performance. The SIR metric gave the same coefficients as the best in-simulation coefficients in the majority of cases. While the coefficient set with the best SIR did not always yield the best BER performance, the differences in performance was minor. The pairwise-optimized 9- and 10-bit coefficients even give slightly better performance than the reference floating-point frequency-domain DBP algorithm, which is possibly caused by the difference in out-of-band response in comparison to the TD-DBP implementation. However, a more detailed investigation of this result is beyond the scope of this work.

Performance with quantized coefficients and quantized signals, with signal requantization points as shown earlier in Fig. 1, was evaluated for the most promising coefficient resolutions (8 and 9 bits). Fig. 5 shows the resulting BER as a function of number of $100-\mathrm{km}$ spans. The performance at 10-bit signal resolution is almost indistinguishable from the floating-point signal case. 9bit signal gives a slight reduction in performance.

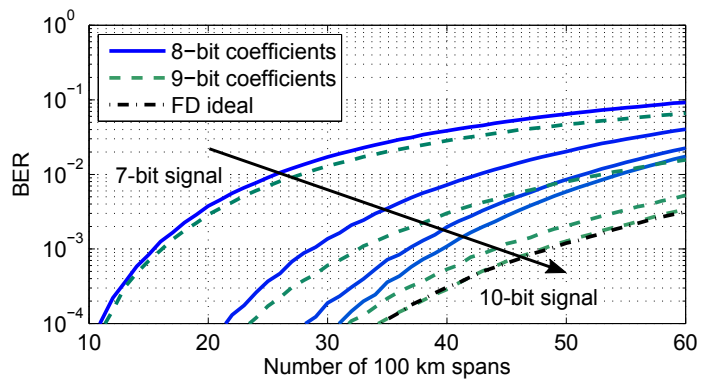

Fig. 5: BER as a function of 100-km spans for 8- and 9-bit coefficients, with 7-10 signal bits, and the frequency-domain (FD) reference algorithm.

\section{Conclusion}

We have presented initial ASIC implementation studies for the Time-Domain Digital Back Propagation algorithm, with the main focus on optimization of the fixed-point-arithmetic FIR filters that dominate the area and power requirements. System performance, measured as number of spans covered at a given BER, is highly dependent on the exact distribution of quantization errors in the FIR-filter coefficients. Previous results ${ }^{1}$ showed that random error perturbations across the filters could improve the reach significantly; here, we actively search for suitable quantized impulse responses, using a metric based on the ISI of the CD-compensated signal, and evaluate the best candidates through system simulation. Alternating two quantized filter versions improves performance further for a given coefficient resolution. For the case studied here, already 9 bits of resolution provides results on par with full-precision floating-point coefficients. With both quantized signal and quantized coefficients, performance close to the floating-point case is achieved.

\section{Acknowledgement}

This work was financially supported by the Knut and Alice Wallenberg Foundation.

\section{References}

[1] C. Fougstedt et al. Time-domain digital back propagation: Algorithm and finite-precision implementation aspects. In Optical Fiber Communication Conference, page W1G.4. Optical Society of America, 2017.

[2] E. Ip and J. Kahn. Compensation of dispersion and nonlinear impairments using digital backpropagation. IEEE J. Lightw. Technol., 26(20):3416-3425, Oct. 2008.

[3] A. Sheikh et al. Dispersion compensation FIR filter with improved robustness to coefficient quantization errors. IEEE J. Lightw. Technol., 34(22):5110-5117, Nov. 2016.

[4] J. Zhang et al. Digital nonlinear compensation based on the modified logarithmic step size. IEEE J. Lightw. Technol., 31(22):3546-3555, Nov. 2013. 